\title{
Avaliar o nível de conhecimento dos profissionais de educação física sobre hipertensão arterial para prescrição de exercício físico
}

\author{
Evaluate physical professionals' level of knowledge about arterial hypertension for prescribing \\ physical exercise \\ Evaluar el nivel de conocimiento de los profesionales de la educación física sobre la hipertensión \\ para la prescripción de ejercicio físico
}

Recebido: 22/09/2021 | Revisado: 28/09/2021 | Aceito: 30/09/2021 | Publicado: 03/10/2021

\author{
Marcos Aurélio da Silva Mendes \\ ORCID: https://orcid.org/0000-0003-0287-7204 \\ Universidade Potiguar, Brasil \\ E-mail: mendesmasm@gmail.com \\ Danilo Costa da Silva \\ ORCID: https://orcid.org/0000-0003-2922-3147 \\ Universidade Potiguar, Brasil \\ E-mail: daniloocosta@ hotmail.com \\ Antônio Eduardo de Oliveira Silva \\ ORCID: https://orcid.org/0000-0002-7149-7152 \\ Universidade Potiguar, Brasil \\ E-mail: eduardohiuga20100@gmail.com \\ Livia Kallahan Soares Pequeno \\ ORCID: https://orcid.org/0000-0001-5453-9245 \\ Universidade Potiguar, Brasil \\ E-mail: liviakallahan@gmail.com \\ Bruna Grandi Fernandes \\ ORCID: https://orcid.org/0000-0003-3803-361X \\ Universidade Potiguar, Brasil \\ E-mail: brunagrandi85@gmail.com \\ Ramile Cristyan Dos Santos \\ ORCID: https://orcid.org/0000-0001-8012-0640 \\ Universidade Potiguar, Brasil \\ E-mail: rcristyans@gmail.com \\ Marcília Ingrid Lima Barroso Nunes \\ ORCID: https://orcid.org/0000-0002-5871-3930 \\ Universidade Potiguar, Brasil \\ E-mail: prof.marciliabarroso@gmail.com \\ Alycia Dannyela de Lima Silva Gobbi \\ ORCID: https://orcid.org/0000-0002-2627-1314 \\ Universidade potiguar, Brasil \\ E-mail: alyciadannyela.al@gmail.com \\ Raille Silva de Jesus \\ ORCID: https://orcid.org/0000-0002-8236-6588 \\ Universidade Potiguar, Brasil \\ E-mail: silvaraille@gmail.com \\ Ana Camila Campelo de Albuquerque Nunes \\ ORCID: https://orcid.org/0000-0002-2030-8331 \\ Universidade Potiguar, Brasil \\ E-mail: Ccampelo32@gmail.com
}

\begin{abstract}
Resumo
O objetivo do presente estudo foi avaliar e descrever o nível de conhecimento dos profissionais de Educação Física sobre as recomendações para prescrição de exercícios físicos para hipertensos em academias de musculação nas zonas Leste e Oeste da cidade de Natal/RN. Trata-se de uma pesquisa do tipo observacional, quantitativa, descritiva com corte transversal. Foram entrevistados 46 profissionais, sendo 41 homens $31.0( \pm 7.2)$ anos, e 5 mulheres $31.2( \pm 5.1)$ anos. Participaram desse estudo profissionais que atuavam em academias da região Leste e Oeste de Natal/RN com no mínimo 5.8 ( \pm 6.6$)$ anos de atuação. Para a análise dos dados foi utilizada a média, assim como o percentual das respostas. Foram incluídos no estudo profissionais devidamente registrados no CREF16/RN. O estudo apresentou resultados onde $91,3 \%$ trabalhavam com hipertensos e que 61,5\% sabiam apenas um critério para aferição da pressão arterial de acordo com a VII Diretriz Brasileira de Hipertensão Arterial. Ainda, apenas 56,5\% conheciam os critérios para hipertensos não
\end{abstract}


iniciarem o exercício naquele dia, e sobre medicamentos, apenas 54,3\% informaram o nome de pelo menos 1 medicamento para controle de hipertensão. Conclui-se, portanto, que o conhecimento desses profissionais para este público foi considerado insuficiente, de acordo com as recomendações da VII Diretriz Brasileira de Hipertensão Arterial e Ciência sobre treinamento para grupos especiais com risco cardiovascular. Sugere-se que mais estudos sejam realizados para se obter dados que auxiliem na elaboração de programas voltados para a atuação profissional, frente a alunos com hipertensão arterial.

Palavras-chave: Hipertensão arterial; Pressão arterial; Educação física e treinamento; Guia de prática clínica.

\begin{abstract}
The objective of the present study was to evaluate and describe the level of knowledge of Physical Education professionals about the recommendations for prescribing physical exercises for hypertensive individuals in weight training gyms in the east and west of the city of Natal / RN. This is an observational, quantitative, descriptive crosssectional research. 46 professionals were interviewed, 41 men $31.0( \pm 7.2)$ years old and 5 women $31.2( \pm 5.1)$ years old. Participated in this study professionals who worked in gyms in the east and west of Natal / RN with at least 5.8 ( \pm 6.6) years of experience. For data analysis, the mean was used, as well as the percentage of responses. Professionals duly registered with CREF16 / RN were included in the study. The study showed results where 91.3\% worked with hypertensive patients and that $61.5 \%$ knew only one criterion for measuring blood pressure according to the VII Brazilian Guideline for Hypertension. Still, only $56.5 \%$ knew the criteria for hypertensive patients not to start exercising that day and about medicines only $54.3 \%$ informed the name of at least 1 of these drugs in hypertension. It is concluded, therefore, that the knowledge of these professionals for this audience was still insufficient according to the recommendations of the VII Brazilian Guideline on Hypertension and science on training for special groups with cardiovascular risk. It is suggested that more studies be carried out to obtain data to assist in the elaboration of programs aimed at professional performance, facing students with arterial hypertension.
\end{abstract}

Keywords: Hypertension; Arterial pressure; Physical education and training; Practice guideline.

\title{
Resumen
}

El objetivo de este estudio fue evaluar y describir el nivel de conocimiento de los profesionales de la Educación Física sobre las recomendaciones para la prescripción de ejercicios físicos para pacientes hipertensos en academias de culturismo en las zonas Este y Oeste de la ciudad de Natal/RN. Se trata de una investigación observacional, cuantitativa, descriptiva y transversal. Cuarenta y seis profesionales fueron entrevistados, 41 hombres $31,0( \pm 7,2)$ años, y 5 mujeres $31,2( \pm 5,1)$ años. El estudio incluyó a profesionales que trabajan en academias en la región Este y Oeste de Natal / RN con al menos $5.8( \pm 6.6)$ años de operación. Para el análisis de los datos se utilizó la media, así como el porcentaje de las respuestas. En el estudio se incluyeron profesionales debidamente registrados en el CREF16/RN. El estudio presentó resultados donde el 91,3\% trabajó con pacientes hipertensos y que el 61,5\% conocía solo un criterio para la medición de la presión arterial según la VII Guía Brasileña de Hipertensión Arterial. Aún así, solo el 56.5\% conocía los criterios para que los pacientes hipertensos no comenzaran a hacer ejercicio ese día, y sobre los medicamentos, solo el $54.3 \%$ informó el nombre de al menos 1 medicamento para el control de la hipertensión. Se concluye, por lo tanto, que el conocimiento de estos profesionales para este público se consideró insuficiente, de acuerdo con las recomendaciones de la VII Guía Brasileña sobre Hipertensión Arterial y Ciencia sobre entrenamiento para grupos especiales con riesgo cardiovascular. Se sugiere que se realicen más estudios para obtener datos que ayuden en el desarrollo de programas orientados al desempeño profesional, frente a estudiantes con hipertensión.

Palabras clave: Hipertensión; Presión arterial; Educación y entrenamiento físico; Guía de práctica clínica.

\section{Introdução}

A Hipertensão Arterial (HA) pode ser conceituada como uma condição clínica multifatorial caracterizada por elevação sustentada dos níveis pressóricos $\geq 140$ e/ ou $90 \mathrm{mmHg}$, e ainda, relata que frequentemente a HA associa-se a distúrbios metabólicos, alterações funcionais e/ou estruturais de órgãos-alvo, sendo agravada pela presença de outros fatores de risco (FR), como Dislipidemia, Obesidade abdominal, intolerância à glicose e Diabetes Mellittus (DM), sendo assim, mantendo associação independente com eventos como morte súbita, Acidente Vascular Encefálico (AVE), Infarto Agudo do Miocárdio (IAM), Insuficiência Cardíaca (IC), Doença Arterial Periférica (DAP) e Doença Renal Crônica (DRC), fatal e não fatal (Sociedade Brasileira de Hipertensão, 2016; Sociedade Brasileira de Cardiologia, 2010).

A Hipertensão Arterial é considerada um problema de saúde pública no Brasil e no mundo (Sociedade Brasileira de Cardiologia, 2010), acometendo $23 \%$ da população adulta brasileira com idade superior a 25 anos, e representa um dos principais fatores de risco para morbidade e mortalidade cardiovasculares, sendo ainda, o fator de risco mais importante para a Cardiopatia 
Isquêmica que acomete 64\% dos pacientes com Infarto Agudo do Miocárdio, além de ser responsável por cerca de $40 \%$ dos casos de aposentadoria precoce e de absenteísmo no trabalho (Negrão et al., 2010; Schmidt, 2011).

Segundo o Sistema de Vigilância de Fatores de Risco e Proteção para Doenças Crônicas por Inquérito Telefônico (VIGITEL), em 2019 a prevalência de Hipertensão autorreferida passou de 22,6\% (2006) para 24,5\%, onde as mulheres ainda continuam com maior prevalência de diagnóstico médico de HA (27,3\%) do que quando comparado aos homens $(21,2 \%)$. No mesmo estudo, foi constatado que na cidade de Natal/RN cerca de $24,5 \%$ de indivíduos são hipertens os, sendo as mulheres $(25,7 \%)$, e os homens $(23,1 \%)$ (VIGITEL, 2019).

O tratamento não medicamentoso (TNM) está cada vez mais utilizado para controle de HÁ. Controle ponderal, medidas nutricionais, como a redução de sódio, prática de atividades físicas (AF), cessação do tabagismo e bebidas alcoólicas, controle de estresse, substituição de anticoncepcionais orais hormonais, redução do peso, entre outros podem reduzir esse quadro clínico (Lubianca et al., 2005; Atthobari et al., 2007; Khitas; Supiano, 2010; Sociedade Brasileira de Hipertensão, 2016). A prática de AF pode ser um caminho para controlar as alterações da pressão arterial (PA), sendo assim, o exercício físico agudo e crônico gera efeitos hipotensores pós-exercícios, desde que sejam planejados e adequados, sempre monitorando volume e intensidade (Negrão et al, 2001; Baster, Baster-Brooks, 2005; Rainforth et al., 2007; Dickinson et al., 2008;).

A prática regular de AF diminui a incidência de HA. Além disso, os hipertensos que alcançam as recomendações de prática de AF para a saúde apresentam uma redução de $27 \%$ a $50 \%$ no risco de mortalidade, mas níveis menores também apresentam efeito benéfico (Fang et al., 2005). No tratamento da HA, benefícios adicionais podem ser obtidos com exercícios físico estruturados, realizando-se o treinamento aeróbico complementado pelo resistido (Sociedade Brasileira de Hipertensão, 2020). Segundo as Diretrizes do American College of Sports Medicine, o treinamento de exercícios aeróbicos leva a reduções na PA de repouso de 5 a $7 \mathrm{mmHg}$ em indivíduos hipertensos, entretanto, podem ser suplementadas com um treinamento contra resistência de intensidade moderada (American College of Sports Medicine, 2013).

Além disso, a AF quando utilizada corretamente e regularmente, como método terapêutico será eficaz no tratamento e no retardo do aparecimento da Hipertensão, sendo assim, é importante a atuação do profissional de Educação Física na prática adequada dos exercícios para que o tratamento seja otimizado (Bezerra, 2011). De acordo com o Conselho Federal de Educação Física - CONFEF, a prescrição de AF necessita da orientação de um Especialista do Exercício. O profissional de Educação Física é capacitado para prescrever e orientar sessões de atividades físicas com fins educacionais, de treinamento, de prevenção de doenças e promoção da saúde (Conselho Federal de Educação Física, 2010).

A presença de pessoas com Hipertensão Arterial nos dias de hoje em academias de musculação na busca por saúde e qualidade de vida melhor é uma realidade, e com isso, o profissional de Educação Física precisa ter capacidade para lidar com este público. Assim, diante das informações coletadas, pode-se verificar que vários profissionais de saúde, principalmente profissionais de Educação Física, podem beneficiar-se de informações, onde grande parte dos atuantes da área não possuem tanto conhecimento em relação ao tema abordado, diante disso, o objetivo do presente estudo foi avaliar e descrever o nível de conhecimento dos profissionais de Educação Física sobre as recomendações para prescrição de exercícios físicos para hipertensos em academias de musculação nas zonas Leste e Oeste da cidade de Natal/RN.

\section{Metodologia}

Trata-se de um estudo descritivo, transversal e quantitativo, realizado no período de setembro a dezembro de 2020, em 26 academias de musculação das zonas Leste e Oeste que se encontram em funcionamento e cadastradas no Conselho Regional de Educação Física - CREF-16, em Natal-RN. A amostra foi composta por 46 profissionais de Educação Física com média de idade $31.0( \pm 6,9)$ anos e escolhidos de forma aleatória, tendo como critério de inclusão ser graduado em Educação Física, estar inscrito regularmente no CREF-16 e trabalhar em academias de musculação cadastradas no mesmo local. Sendo assim a idade 
média correspondeu a, 5 mulheres com 31.2 ( \pm 5.1$)$ anos, e 41 homens com 31,0 ( \pm 7.2$)$ anos, independentemente da idade, tempo de formação profissional e que estivessem presentes no momento da visita à academia.

Para esta pesquisa foi utilizado como instrumento de coleta um questionário com nove questões fechadas e abertas elaborado e aplicado de forma presencial nas academias, e, devido a pandemia do COVID-19, foi elaborado um questionário com as mesmas questões de forma on-line através do Google Forms no intuito de diminuir o contato entre os entrevistados e o entrevistador, onde era enviado aos voluntários através do WhatsApp ou e-mail. As nove questões utilizadas tiveram o objetivo de verificar o perfil de conhecimento sobre HA através de perguntas objetivas e subjetivas de características gerais e formação profissional, sobre o conhecimento e critérios de HA, tipos de exames laboratoriais solicitados, cuidados gerais para a prática de atividade física e formas de acompanhamento durante o treinamento, de acordo com a VII Diretriz Brasileira de Hipertensão $\operatorname{Arterial}^{1} 1$ ( $7^{\mathrm{a}}$ Diretriz Brasileira de Hipertensão Arterial, 2016).

Inicialmente foi realizado um levantamento das academias de musculação cadastradas nas zonas Leste e Oeste. A partir destes dados foram visitadas todas essas academias, sendo solicitada a autorização do coordenador ou do professor responsável pelo estabelecimento. Estas visitas ocorreram nos horários de funcionamento, de forma aleatória. Os questionários foram entregues aos profissionais no momento da visita, sob a supervisão dos pesquisadores que permaneceram no local até que os profissionais respondessem por completo, e aqueles que optaram pelo formulário on-line os pesquisadores solicitaram retorno imediato após término no intuito de diminuir o tempo para verificação das respostas. Todos os participantes assinaram um Termo de Consentimento Livre e Esclarecido (TCLE), concordando em participar do estudo. Os dados foram processados no programa EXCEL versão 2013 e tratados em análise descritiva (frequência, média e desvio padrão da média).

\section{Resultados e discussões}

A Tabela 1 demonstra as características dos profissionais de Educação Física nas academias de musculação das zonas Leste e Oeste de Natal/RN. A idade de todos os praticantes de musculação foi em média de 31,0 ( $\pm 7,0)$ anos, e que no total de 46 profissionais, $19(41,3 \%)$ possuem pós-graduação, sendo, apenas 6 (13\%) em grupos especiais e $19(41,3 \%)$ em outras especialidades, e também, 42 (91,3\%) profissionais já trabalharam ou trabalham com alunos hipertensos e para 14 (30,4\%) do total, o parâmetro utilizado para diagnosticar a HA veio através da informação do próprio aluno, e 32 (69,6\%) profissionais, responderam solicitar exames médicos para alunos hipertensos.

\footnotetext{
${ }^{1}$ VII Diretriz Brasileira de Hipertensão Arterial - trabalho realizado pela Sociedades Brasileira de Cardiologia (SBC), Sociedade Brasileira de Hipertensão (SBH) e Sociedade Brasileira de Nefrologia (SBN) com objetivo de orientar os profissionais de saúde quanto às medidas preventivas e a atenção aos portadores de hipertensão arterial, visando reduzir as complicações da doença, considerado o mais expressivo fator de risco para as moléstias cardiovasculares.
} 
Tabela 1. Características dos profissionais de Educação Física das academias da zona Leste e Oeste de Natal/RN.

\begin{tabular}{|c|c|}
\hline CARACTERÍSTICAS & $\mathbf{n}(\mathbf{m} \pm \mathrm{dp})$ \\
\hline $\mathbf{N}$ & 46 \\
\hline Idade (anos) & $31,0 \pm 7,0$ \\
\hline Masculino, $\mathrm{n}$ & $41(31,0 \pm 7,2)$ \\
\hline Feminino, $\mathrm{n}$ & $5(31,2 \pm 5,1)$ \\
\hline Formação (anos) & $5.8 \pm 6,6$ \\
\hline Pós Graduação SIM, n (\%) & $19(41,3)$ \\
\hline \multicolumn{2}{|l|}{ Pós Graduação - Tipo } \\
\hline Grupos especiais n (\%) & $6(13)$ \\
\hline Outra n $(\%)$ & $13(28,3)$ \\
\hline Não possui n $(\%)$ & $27(58,7)$ \\
\hline \multicolumn{2}{|l|}{ Já treinou ou treina alunos com $\mathrm{HA}$} \\
\hline $\operatorname{Sim} \mathrm{n}, \%$ & $42(91,3)$ \\
\hline Não n, \% & $4(8,7)$ \\
\hline Não sabe $n, \%$ & $0(0)$ \\
\hline \multicolumn{2}{|l|}{ Diagnóstico de HA n (\%) } \\
\hline Informação próprio aluno n, \% & $14(30,4)$ \\
\hline Solicitação de exames n, \% & $32(69,6)$ \\
\hline
\end{tabular}

Fonte: Dados da pesquisa.

O Gráfico 1, mostra que, 30,4\% dos 46 profissionais não solicitaram nenhum dos critérios essenciais para diagnóstico da Hipertensão Arterial, sendo eles: eletrocardiograma, teste ergométrico, mensuração da pressão arterial, exames essenciais para diagnóstico clínicos, e 19,6\% dos profissionais solicitaram os três tipos de exames. A avaliação clínica inicial a um programa de atividade física solicitada pelo profissional de Educação Física, é essencial para a realização da sua prescrição principalmente da intensidade e volume do treinamento e por meio do exame clínico pode-se avaliar sinais e sintomas, ou outras condições clínicas e patológicas associadas ao critério de diagnóstico para Hipertensão, ao qual se faz importante o conhecimento adequado na Hipertensão (Nunes et al., 2020).

Gráfico 1. Percentuais de respostas sobre o tipo de exame solicitado ao hipertenso.

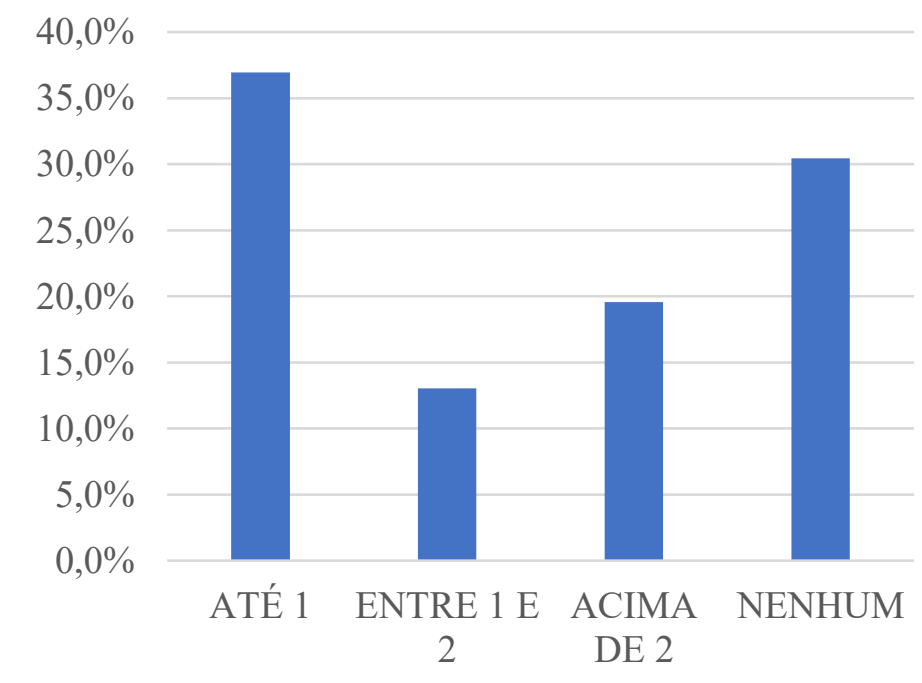


Gráfico 2. Percentuais de respostas sobre nível de PA para o diagnóstico de Hipertensão.

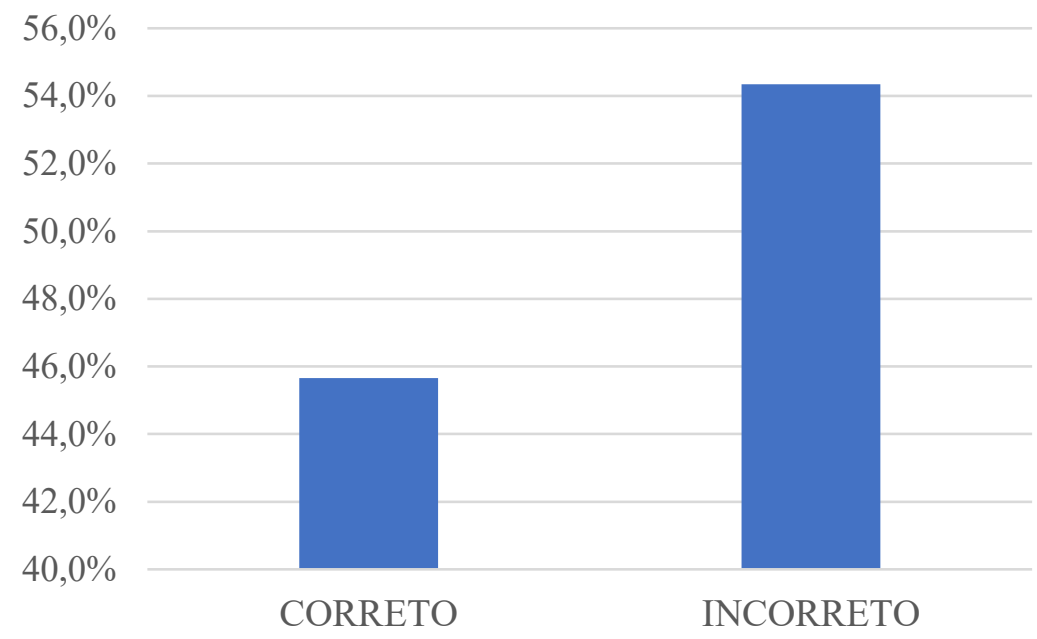

Fonte: Dados da pesquisa.

Pode-se verificar na pesquisa que dos 46 profissionais, 21 (45,7 \%) responderam a opção correta $(140 / 90 \mathrm{mmHg})$, e, quando comparado ao estudo de Nunes et al., (2020) pouco mais da metade, 51\%, conseguiu êxito nesse tipo de questão. De acordo com a Diretriz Brasileira de Hipertensão Arterial, 2020, a Hipertensão é caracterizada por elevação persistente da pressão arterial (PA), ou seja, PA sistólica (PAS) maior ou igual a 140 mmHg e/ou PA diastólica (PAD) maior ou igual a 90 mmHg, medida com a técnica correta, em pelo menos duas ocasiões diferentes, na ausência de medicação anti-hipertensiva. É primordial que o profissional de Educação Física que trabalha com grupos especiais, principalmente hipertensos, possua o conhecimento do diagnóstico do nível de pressão arterial considerado estágio I para Hipertensão (Nunes et al., 2020).

No Gráfico 3, ao analisar se os profissionais aferiam a PA antes e após o treinamento, o resultado apontou que 56\% do total aferia em um destes momentos e/ou em ambos, o que corrobora quando comparado com o estudo de Verissímo (2017), que mostrou que 30,8\% realizam aferição da PA antes e após o treino.

Gráfico 3. Percentuais de respostas sobre verificação da PA antes e após o treinamento.

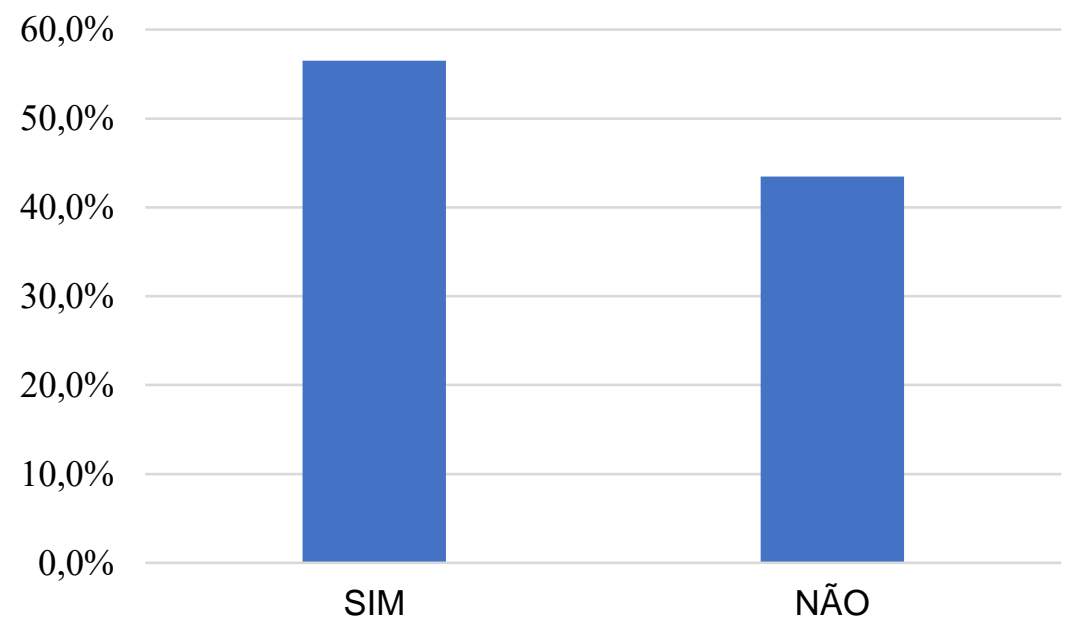

Fonte: Dados da pesquisa. 
Ainda, essa pesquisa mostrou que dos 46 profissionais de Educação Física, 26 (56,5\%) destes responderam que realizam aferição da PA e utilizam algum método de mensuração da PA, e que apenas 16 (61,5\%) responderam que conheciam apenas um critério para mensurar a PA (gráfico 4) e a escolha desses critérios variavam em respostas diferentes, como: utilização do aparelho de esfigmomanômetro, pulsação do punho ou informação do aluno. Além disso 20 (43,5\%) profissionais do total da pesquisa, responderam que não realizam aferição da PA, onde $10(50 \%)$ destes profissionais (Gráfico 5) informaram que a falta do esfigmomanômetro nas academias torna-se um dos principais impasses para aferição da PA.

Quando comparado com os estudos de Nunes et al. (2020), onde mostra que 72\% conheciam apenas um critério para mensurar a pressão arterial e $61 \%$ dos profissionais informam que a falta do esfigmomanômetro nas academias torna-se um dos principais problemas para aferição da PA, entende-se, que a não mensuração da PA ocorre pela falta de conhecimento dos critérios associada a falta de aparelhos específicos nas academias, e consequentemente pode-se ter um aumento do risco de acidentes relacionados a elevação acentuada da PA no hipertenso e até leva-lo a óbito durante o treinamento (Nunes et al., 2020).

Gráfico 4. Percentuais de respostas sobre critérios para aferição da PA de alunos hipertensos.

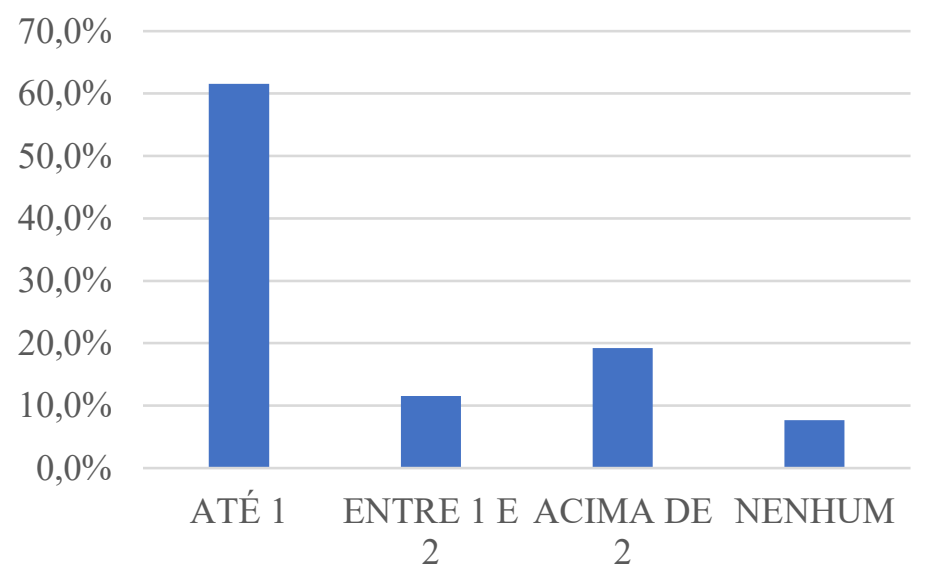

Fonte: Dados da pesquisa.

Gráfico 5. Percentuais de respostas relacionadas a motivos para a não medição da PA do hipertenso.

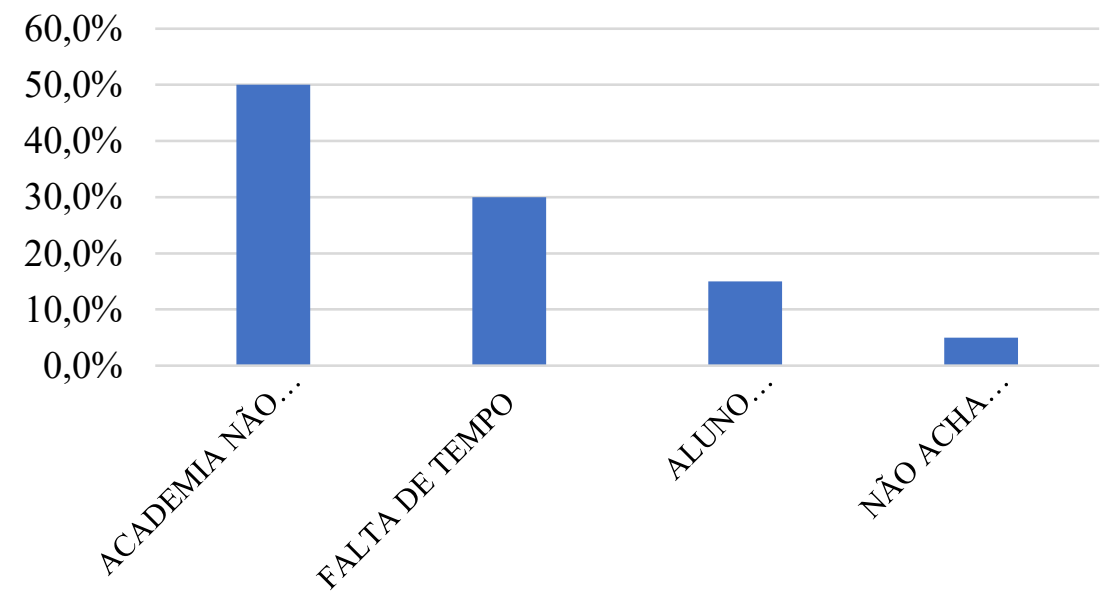

Fonte: Dados da pesquisa.

De acordo com a Diretriz de Hipertensão Arterial, 2020, a sessão de treinamento não deve ser realizada se a PA estiver acima de 160/105 mmHg, e recomenda medir a PA durante o exercício aeróbico em hipertensos hiper-reativos e diminuir a 
intensidade se ela estiver acima de 180/105 mmHg (Sociedade Brasileira de Hipertensão, 2020). Com isso o profissional de Educação Física deve realizar a verificação da PA antes da realização de qualquer atividade física para os alunos hipertensos, pois o aumento da pressão arterial sistólica e da frequência cardíaca, gera um aumento do duplo produto, e este, se maior que 30 bpm mmHg oferece alto risco de Acidente Vascular Cerebral, Embolia Pulmonar, Infarto Agudo do Miocárdio e/ou outras complicações inerentes ao sistema cardiovascular tanto em pessoas saudáveis e ainda com risco elevado em pessoas hipertensas (Nunes et al., 2020).

O Gráfico 6, mostra o conhecimento dos profissionais sobre os níveis de pressão sistólica e diastólica em específico e o resultado foi que, 56,5\% conheciam essa informação e, entendendo que se os valores estivessem 160 e/ou $105 \mathrm{mmHg}$ antes do treinamento, o profissional não permitiria que o aluno treinasse normalmente, adotando procedimento correto de conduta segura para este público.

Gráfico 6. Percentuais de respostas sobre medidas de PA para que o aluno não inicie o exercício no dia da prática da atividade física.

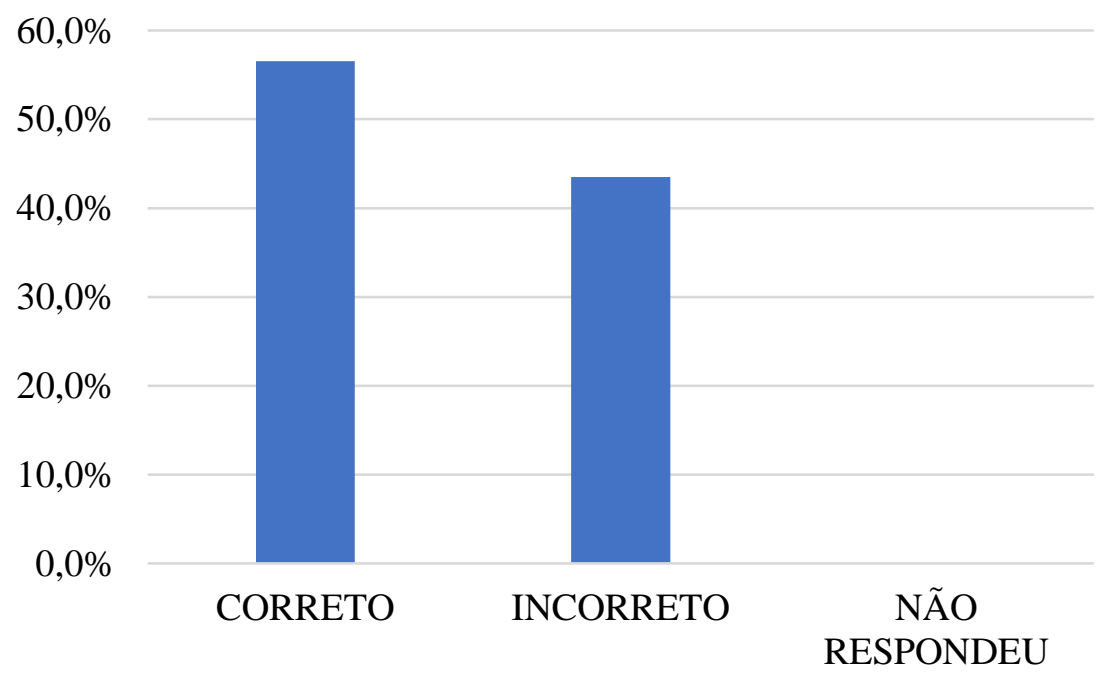

Fonte: Dados da pesquisa.

Resultados (Gráfico 6) antagônicos ao demostrando por Monteiro et al. (2010) quando, ao aplicar um questionário sobre o conhecimento dos profissionais quanto às situações que contraindicavam a atividade física em hipertensos, a maioria, 43.1\%, respondeu que a pressão arterial elevada seria uma situação para contraindicar. Já em estudo de Nunes et al. (2020) foi pesquisado o conhecimento dos profissionais sobre os níveis de pressão sistólica e diastólica, em específico, e o resultado foi que 65\% não conheciam essa informação. Ao comparar os dois estudos têm-se como conclusão de que os profissionais de Educação Física não têm conhecimento para iniciar programas de treinamento para pacientes hipertenso.

Além disso, de acordo Nieman (2011) a intensidade do treino aeróbio em hipertensos pode ser determinada através da escala de BORG modificada para esse fim. A resposta acima corresponde correto (utiliza a escala de BORG). O gráfico 7 mostra que, $97,8 \%$ dos profissionais voluntário tinham total conhecimento dessa escala de fácil aplicabilidade para esse público e quando comparado com os estudo de Nunes et al. (2020), os resultados são totalmente antagônico, onde mostram que $78 \%$ dos profissionais não conheciam essa escala, e ainda, com o estudo Veríssimo e Medeiros (2017) 62\% dos profissionais entrevistados responderam a alternativa incorreta, assim, depois da análise destes dados, pode-se observar se os profissionais tem buscado ter mais conhecimento da utilização deste método no intuito de melhorar o seu desempenho. 
Gráfico 7. Percentuais de respostas sobre a melhor maneira de acompanhar o esforço durante o treinamento aeróbio.

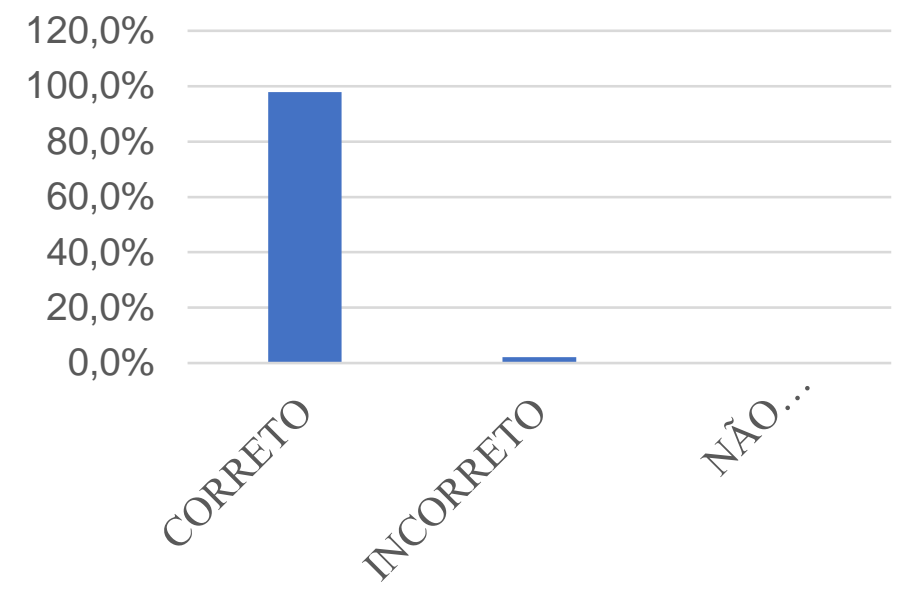

Fonte: Dados da pesquisa.

O Gráfico 8 mostra o resultado referente a utilização da manobra de Valsalva durante o treinamento resistido. A resposta acima corresponde correto (evitar manobra Valsalva), e de acordo com a Diretriz do Americam College of Sports Medicine (2013), deve-se evitar a manobra de Valsalva durante o treinamento contra resistência. Na pesquisa a resposta foi bastante satisfatória, onde $93,5 \%$ dos profissionais mostraram conhecimento acerca desse critério. Quando comparado com o de Nunes et al. (2020), 80\% dos profissionais mostraram conhecimento acerca desse critério; e o estudo de Veríssimo e Medeiros (2017) os números foram inferiores, onde $88 \%$ também conseguiram responder corretamente, ou seja, um bom nível de conhecimento do profissional nesse aspecto pode reduzir riscos de picos hipertensivos neste público.

Gráfico 8. Percentuais de respostas sobre o que fazer para evitar a manobra de Valsalva.

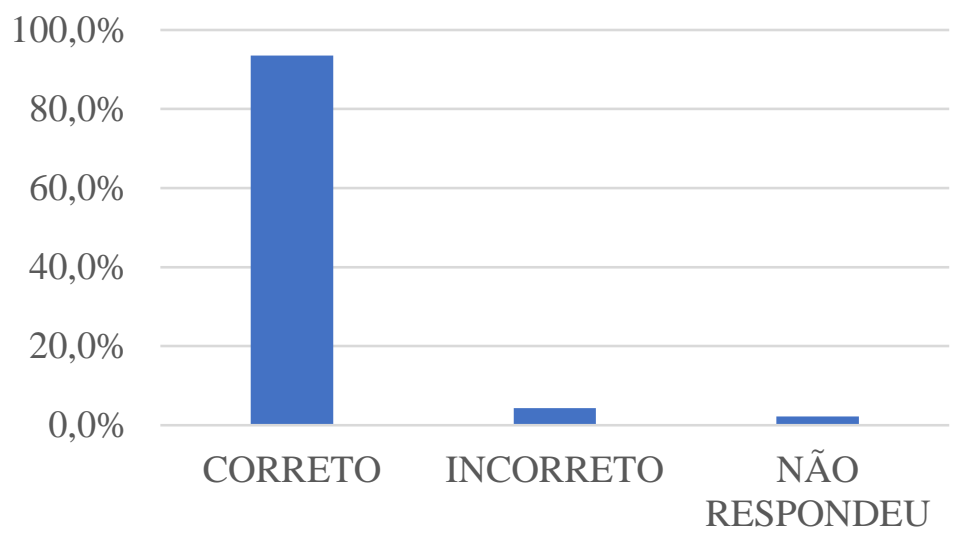

Fonte: Dados da pesquisa.

Por fim, há muitos medicamentos que são eficientes para o tratamento da maioria dos pacientes que podem precisar tomar pelo menos dois medicamentos para alcançar os níveis desejados de PA (American College of Sports Medicine, 2013). O profissional de Educação Física atuante com público de Grupos Especiais têm a necessidade de conhecimento sobre tipos de fármacos que possam alterar o rendimento no tocante a prática de atividade física de hipertenso, sendo assim, a prescrição de exercícios para esse público deve ser feita com muita cautela e um dos cuidados a ser tomado na anamnese é também quanto à utilização de medicamentos anti-hipertensivos, para que se possa avaliar os riscos e saber com qual intensidade será realizado o treinamento. 
No presente estudo mais da metade dos profissionais $(54,3 \%)$ soube informar pelo menos um desses medicamentos e apenas (19,6\%) não soube informar qualquer tipo de medicamento relacionado ao controle da Hipertensão (gráfico 9). Dentre os mais citados estão: Losartana, Captopril, os betabloqueadores e os diuréticos, anti-hipertensivos como alfa-bloqueadores, bloqueadores de canais de cálcio e vasodilatadores. De acordo com Vanzelli et al. (2005) esses medicamentos, principalmente os betabloqueadores, reduzem a frequência cardíaca máxima e de repouso, e o uso contínuo, pode interferir na determinação da intensidade a ser aplicada no exercício e consequentemente o não respeito da individualidade do aluno em relação a carga de treino.

Gráfico 9. Percentuais de repostas sobre quais medicamentos ou classes destes são mais utilizados para o controle da pressão arterial.

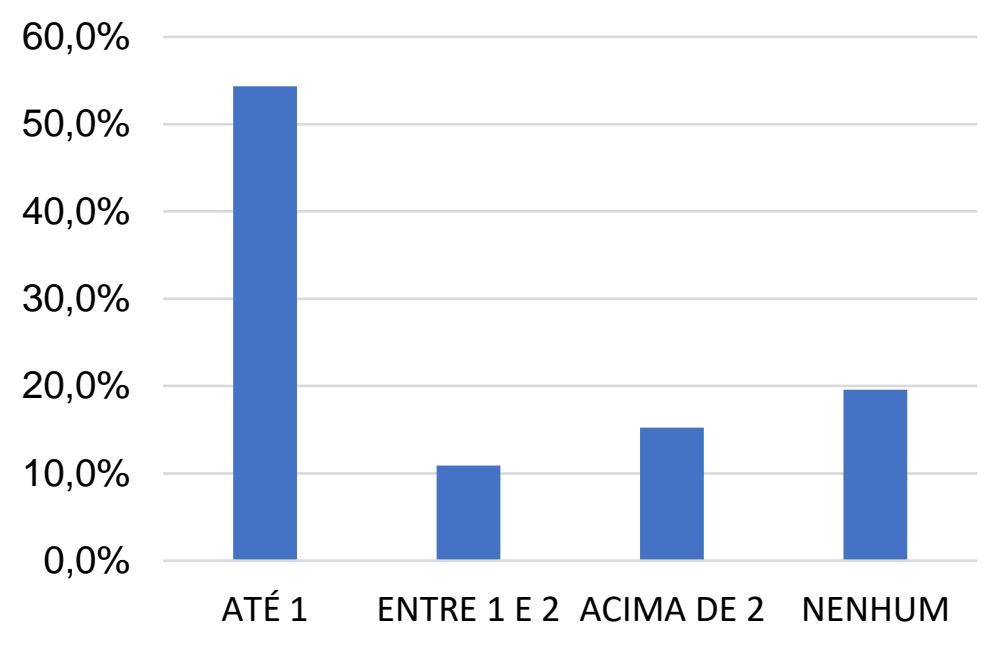

Fonte: Dados da pesquisa.

Os betabloqueadores, principalmente os de tipo cardíaco não seletivos, podem reduzir a capacidade de exercício máxima e submáxima principalmente em pacientes sem Isquemia miocárdica, (American College of Sports Medicine, 2013; Netto, 2017). Medicamentos anti-hipertensivos, como alfa-bloqueadores, bloqueadores de canais de cálcio e vasodilatadores, podem levar a reduções excessivas súbitas na PA após o exercício. O profissional deve entender e monitorar cuidadosamente o período de resfriamento desses indivíduos. Há muitos fármacos eficientes para o tratamento de Hipertensão, mas a maioria dos pacientes pode precisar tomar pelo menos dois medicamentos para alcançar os níveis desejados de PA (American College of Sports Medicine, 2013).

\section{Conclusões}

Nesta pesquisa foi observado que os profissionais apresentaram um conhecimento um pouco melhor em comparação com outros estudos na região Norte de Natal/RN (Nunes et al., 2020), mas ainda não é o suficiente em relação as práticas de manejo com hipertensos, conforme as recomendações da VII Diretriz Brasileira Hipertensão Arterial e Ciência sobre treinamento para Grupos Especiais com risco cardiovascular como: critérios da Hipertensão Arterial, cuidados gerais com o hipertenso para a prática de atividade física e formas de acompanhamento desse hipertenso durante o treinamento.

Os profissionais atuantes com esse público deve estar constantemente buscando mais conhecimento especializado e não só dispor de conhecimentos adquiridos na graduação, sendo assim, deve-se estimular os donos de academias que capacitem seus profissionais para que haja um atendimento mais completo e seguro, para que não ocorram situações indesejáveis em suas 
academias de musculação. Além disso, as instituições de ensino também devem dar uma atenção especial para disciplinas da área do treinamento físico para grupos em situações diferenciadas, como os hipertensos, diabéticos, idosos e obesos, e proporcionar a vivência clínica para os estudantes da graduação a fim de desenvolver qualificações de intervenção (Gomes; Gomes, 2012).

Sugere-se mais investigações acerca do conhecimento dos profissionais de Educação Física para prescrição de treinamento de indivíduos acometidos de Hipertensão Arterial para obter-se dados que auxiliem na elaboração de alternativas voltadas para a formação e atuação profissional frente a alunos com HA, tendo em vista o crescente número de hipertensos no país e nesta região em específico.

\section{Referências}

Atthobari, J. et al. (2007). The impact of hormonal contraceptives on blood pressure, urinary albumin excretion and glomerular filtration rate. British Journal of Clinical Pharmacology, 63(2), 224-31.

Baster, T. \& Baster-Brooks, C. (2005). Exercise and hypertension. Australian Family Physician, 34(6), 419-24.

Brasil. (2019) Vigilância de fatores de risco e proteção para doenças crônicas por inquérito telefônico. Ministério da Saúde, Brasília, DF.

CONFEF (2010). Estatuto do Conselho Federal de Educação Física - CONFEF. Diário Oficial, 237(1), 137-43. https://www.confef.org.br/confef/conteudo/471.

Dickinson, H. O. et al. (2008). Relaxation therapies for the management of primary hypertension in adults. Cochrane Database Syst. Rev., 23(1).

Fang, J. et al. (2005). Exercise and cardiovascular outcomes by hypertensive status: NHANES I epidemiological follow-up study, 1971-1992. American Journal of Hypertension, 18(6), 751-75.

Gomes, L. M, \& Gomes, L. M. (2012) A importância do profissional de educação física na melhoria da qualidade de vida de diabéticos e hipertensos. IV Congresso Sudeste de Ciências do Esporte, Vitória, ES. https://cienciadotreinamento.com.br/wp-content/uploads/2017/06/EDUCA\%C3\%87\%C3\%83OF\%C3\%8DSICA-IDENTIDADES-E-CAMPOS-DE-ATUA\%C3\%87\%C3\%83O..pdf.

Júnior, P. C. S. da S. et al. (1999). Os efeitos da atividade física na prevenção da hipertensão. Revista Brasileira de Medicina do Esporte, 5(2). https://www.scielo.br/j/rbme/a/HXpP6HJYtgFm4VRSSzmcDWn/abstract/?lang=pt.

Kithas, P. A, \& Supiano, M. A. (2010) Practical recommendations for treatment of hypertension in older patients. Vasc Health Risk Manag., 6(9), 561-69.

Lubianca, J. N. et al. (2005). Stopping oral contraceptives: an effective blood pressure-lowering intervention in women with hypertension. Journal of Human Hypertension, 19(6) 451-55.

Negrão, C. E. et al. (2010). Cardiologia do exercício do atleta ao cardiopata. Editora Manole, 3, 451.

Netto, R. O. R. F. et al. (2017). Efeitos colaterais dos anti-hipertensivos mediante a prática da atividade física. Revista Magsul de Educação Física na Fronteira, 2(3). http://bibmagsul.kinghost.net/revista2016/index.php/RevMagEdFis/article/viewFile/411/300.

Nunes, A. C. C. A. et al. (2020). Nível básico de conhecimento do profissional de educação física sobre treinamento para hipertensos. Brazilian Journal of Development, 6(11), 91083-96. https://www.brazilianjournals.com/index.php/BRJD/article/view/20348.

Rainforth, M. V. et al. (2007). Stress reduction programs in patients with elevated blood pressure: a systematic review and meta-analysis. Curr. Hypertens. Rep., $9(6), 520-28$.

Schmidt, M. I. et al. (2011). Doenças crônicas não transmissíveis no Brasil: carga e desafios atuais. The Lancet, 377(9781), 1949-61.

Sociedade Brasileira De Cardiologia. (2010). VI Diretrizes Brasileiras de Hipertensão. Arquivos Brasileiros de Cardiologia, 95(1), 1-51.

Sociedade Brasileira De Hipertensão. (2020). Diretrizes de Hipertensão Arterial. Brazilian Guidelines of Hypertension.

Sociedade Brasileira De Hipertensão. (2016). VII Diretriz Brasileira de Hipertensão Arterial. Revista Hipertensão Arterial, 19(4), 10.

Sociedade Brasileira De Hipertensão. (2016). VII Diretriz Brasileira de Hipertensão Arterial. Revista Hipertensão Arterial, $19(4), 47$.

Veríssimo E. M, \& Medeiros J. A. (2017). Perfil do Conhecimento do profissional de educação física sobre hipertensão nas academias de musculação. Revista $U N I-R N, 16(17) 257-73$. 